Transgenic animals

New advances in the field

\author{
from Robin H. Lovell-Badge
}

INJECTION of new genetic material into fertilized mouse eggs followed by development of the eggs into transgenic mice in foster mothers has become an almost routine tool in the analysis of a wide range of biological problems ${ }^{1}$. If the same technique could be used for farm animals it could be of great practical and economic importance, allowing the transfer of characteristics from one breed to another much more rapidly than by conventional breeding. More dramatically, it could allow genetic transfer between species. The paper by Hammer et al. ${ }^{2}$ on page 608 of this issue is the first demonstration that it is indeed possible to use microinjection to obtain integration into the genome, and in some cases expression, of exogenous DNA in large mammals - namely rabbits, pigs and sheep.

To work with large domestic animals rather than mice requires a considerable investment, particularly in the number of animals required. While it is normal to obtain up to 40 eggs from a single superovulated mouse, a ewe gives on average only three or four. Reimplantation of manipulated embryos may pose an even greater problem. Even though most will not survive, mice can tolerate up to 20 offspring, whereas sheep and cattle will not give birth to more than two. The occurrence of freemartins would be an additional problem with cattle.

These considerations have presumably influenced the selection of species used by Hammer et al. Pigs offer advantages because they are multiparous, sheep are an example of a difficult species and rabbits, while a reasonably convenient laboratory animal (and many a farmer's nightmare), are similar to pigs and sheep in many aspects of their early embryonic development. It is also essential to choose techniques that are efficient. It is clear from microinjection of tissue culture cells ${ }^{3}$ and mouse embryos that transformation frequency is very low unless DNA is injected into the nucleus. But the eggs of many domestic animals have such an opaque cytoplasm that it is impossible to see their pronuclei or nuclei with conventional light microscopy. DNA-specific fluorochromes have been used to visualize nuclei in early bovine embryos ${ }^{4}$, but the damage induced by ultraviolet light can severely compromise their development.

For sheep embryos, Hammer and colleagues have solved the problem to a large extent (but see below) simply by the use of interference contrast microscopy. More drastic procedures were necessary for the $\mathrm{pig}^{5}$. Centrifugation of the embryos at $15,000 \mathrm{~g}$ for three minutes separates the

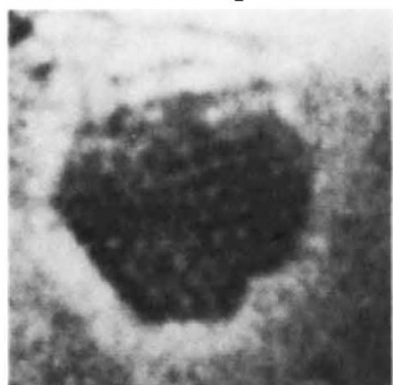

cytoplasm into three layers. Most droplets and granules, corresponding to the lowest buoyant density but greatest optical density, are found at one pole. The equatorial region contains a band of small clear droplets and the remaining half of the zygote has a translucent appearance. Nuclear structures may be found in either of these regions and are therefore clearly visible. The centrifugation of the pig embryos seems not to affect their subsequent development. It may also be a useful technique for other species, notably cattle, to allow experiments involving nuclear transfer for investigating the possibility of cloning ${ }^{6}$, or parthenogenic development ${ }^{7}$, of farm animals.

The gene chosen by Hammer et al. for injection into the nuclei of embryos of all three species is the mouse metallothionein-human growth hormone fusion gene that has been used in the same laboratory (Ralph Brinster's) for experiments with mice ${ }^{8,9}$. In each case about ten per cent of the injected embryos survived to term after reimplantation into foster mothers. The frequencies of fusion gene integration in the rabbit $(12.8 \%)$ and pig $(11.0 \%)$, although lower than the overall frequencies in mice (about 27\%), are good, and other workers using these species will be much encouraged.

By contrast, the results with sheep are disappointing. Only one out of the 73 foetuses or newborn carried the injected fusion gene and even then it was obviously deleted or rearranged. It is conceivable that sheep embryos lack the appropriate mechanisms that fortuitously integrate exogenous DNA, but it seems more likely that some aspect of the technique needs refining. Perhaps more copies need to be injected and perhaps the visualization of the pronuclei needs to be improved (nuclear structures were not seen in a proportion of fertilized sheep eggs and these may correspond to more 'receptive' stages). Otherwise it may be necessary to adopt alternative strategies such as the use of retroviral gene vectors that are just beginning to work with mice.

Ovulation and fertilization are not v ry synchronous in the rabbit, pig and sheep, so it was necessary for Hammer et al. to inject embryos that ranged from 1-cell to 4-cell stages. For mice, even when the injections were at the pronuclear 1-cell stage, some of the transgenic animals were mosaic, presumably because gene integration was delayed. When this mosaicism was in the germline, few offspring inherited the introduced gene sequence ${ }^{10}$.

\title{
Japanese gold in atomic motion
}

Use of a high-resolution electron mircoscope and video recorder has allowed the first real-time recordings of the motion of individual atoms. The pictures above show an ultra-fine particle of gold (18 $\AA$ across and containing about 500 atoms) photographed using the new technique by Sumio Iijima, a group leader in Japan's ERATO ultra-fine particles project (see Nature 305, 373; 1983). It can be seen that the shape of the particle changes over time, in discrete steps from top left to bottom right with all the gold atoms making up the ultra-fine particle moving cooperatively to form various crystalline structures. The gold atoms in ultra-fine particles thus seem to behave in a 'quasi-solid' manner with some properties like those of a liquid —one gold particle can even 'swallow-up' a smaller one. These phenomena await theoretical explanation.
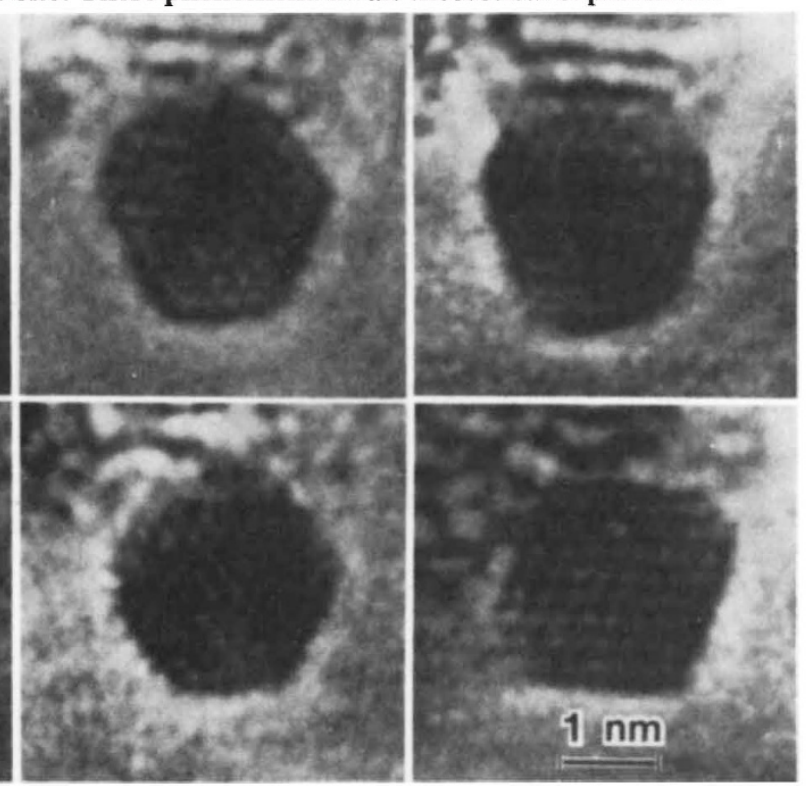

๑)1985 Nature Publishing Group 